\title{
Rational expectations in limited dependent variable models
}

\author{
Lung-fei Lee \\ Department of Economics, Lorch Hall, The University of Michigan, 611 Tappan Street, Ann Arbor, MI 48109, \\ USA
}

Received 2 August 1993; accepted 14 March 1994

\begin{abstract}
The appropriate characterizations of rational expectations solutions in one-limit and two-limit models are derived. We prove the existence and uniqueness of the rational expectations solution for each model. Our analysis is gencral without imposing serial independence and the parametric distributional assumption.
\end{abstract}

JEL classification: $\mathrm{C} 24 ; \mathrm{C} 62$

\section{Introduction}

The problem of rational expectations in limited dependent variable models was first briefly discussed in Chanda and Maddala (1983). The usefulness of rational expectations limited dependent variables models in empirical studies can be found in Shonkwiler and Maddala (1985) and Holt and Johnson (1989) for agricultural commodities markets with price supports and Pesaran and Samiei (1992) for models of exchange rate determination under a target zone. There are some debates on appropriate specifications and estimation of such models (Maddala, 1990; Pesaran, 1990; and Donald and Maddala, 1992). Pesaran (1990) and Pesaran and Samiei (1992) indicated that a kink was incorrectly introduced in expected price functions in Shonkwiler and Maddala (1985) and Maddala (1990). Pesaran and Samiei have formulated a rational expectations solution equation and proved analytically the existence of a unique rational expectations solution in such models. With the existence of a unique rational expectations solution, Pesaran and Samiei have suggested an elegant full information maximum likelihood method for the estimation of such models. While the estimation method and the empirical application in Pesaran and Samiei (1992) are very interesting, the formulation of the rational expectations solution does not completely correct the errors in the literature. The shortcoming was finally corrected in Donald and Maddala (1992). Sufficient conditions for the existence and uniqueness of rational expectations are provided.

However, the analysis by Donald and Maddala (1992) does not fully take account of the 
change in the characterization of the rational expectations solution. The proof in Donald and Maddala followed closely the arguments in Pesaran and Samiei (1992). It suggests that the same conditions in Pesaran and Samiei (1992) are needed for the existence and uniqueness of the rational expectations solution. In this paper we provide a more general analysis with less restrictive assumptions on the economic and stochastic structures of the models. We provide an appropriate characterization of the rational expectations solution, which is more general than the characterization in Donald and Maddala (1992). Contrary to the existence and uniqueness analyses in Pesaran and Samiei (1992) and Donald and Maddala (1992) that assume serially uncorrelated and normal distributed disturbances, our analysis is quite general. There is neither serial independence nor specific parametric distributions imposed on the disturbances. This paper clarifies the general issues on the existence and uniqueness of the rational expectations in limited dependent variables models. ${ }^{1}$ It provides the appropriate formulas for the computation of rational expectations solutions, which are important for the empirical estimation of such models.

\section{Rational expectations in limited dependent variables models}

Limited dependent variable models were introduced in econometrics in Tobin (1958) (see also Amemiya, 1973). Consider first the one-limit model with expectations. Let

$$
p_{t}^{*}=\gamma p_{t}^{\mathrm{e}}+\beta^{\prime} x_{t}+u_{t}
$$

be the latent equation defined before censoring. The observed dependent variable (market price for the price support model) is

$$
p_{t}=\max \left\{p_{t}^{*}, p_{t l}\right\}
$$

where $p_{t l}$ is the lower bound (support price). Let $\mathscr{I}_{t-1}$ be the information set available at time $t-1$ when the expectation $p_{t}^{\mathrm{e}}$ of $p_{t}$ is formed. The bound $p_{t l}$ is fully credible and is in the agent's information set $\mathscr{F}_{t-1}$. As in Pesaran and Samiei (1992), we consider the realistic situation that the agent at time $t-1$ does not know whether $p_{t}$ is above or below $p_{t l}$. Mathematically, let $I_{t}$ be the dichotomous indicator:

$$
I_{t}= \begin{cases}1, & \text { if } p_{t}^{*} \leqslant p_{t l} \\ 0, & \text { if } p_{t}^{*}>p_{t l}\end{cases}
$$

Thus, $I_{t}=1$ indicates that the support price is the observed price in the price support model. The situation considered is that $I_{t}$ is not in the information set $\mathscr{I}_{t}{ }^{2}$. In addition to $p_{t}^{\mathrm{e}}$, when $x_{t}$ is not perfectly predictable, the agent is assumed to form the rational expectation $x_{t}^{\mathrm{e}}$ of $x_{t}$ at time $t-1$. As in Muth (1961), we assume that the agent's expectation is rational in that the expected price is an unbiased prediction of the actual price. Therefore,

\footnotetext{
${ }^{1}$ Our analysis has recently been successfully generalized in Pesaran and Ruge-Murcia (1993) to more complicated models with stochastic thresholds.

${ }^{2}$ Pesaran (1990) has argued that Maddala (1990) had implicitly assumed that $I_{t}$ were in $\mathscr{I}_{t-1}$ in his formulation.
} 


$$
p_{t}^{\mathrm{e}}=\mathrm{E}\left(p_{t} \mid \mathscr{I}_{t-1}\right)
$$

and $x_{t}^{\mathrm{e}}=\mathrm{E}\left(x_{t} \mid \mathscr{I}_{t-1}\right)$.

With the model specified, we consider the characterization of the rational expectations solution of the model. Let $v_{t}=x_{t}-\mathrm{E}\left(x_{t} \mid \mathscr{I}_{t-1}\right)$ be the prediction error of $x_{t}$. It follows from (2.3) that $I_{t}=1$ if and only if $w_{t} \leqslant p_{t i}-\gamma \mathrm{E}\left(p_{t} \mid \mathscr{I}_{t-1}\right)-\beta^{\prime} \mathrm{E}\left(x_{t} \mid \mathscr{I}_{t-1}\right)$, where $w_{t}=u_{t}+\beta^{\prime} v_{t}$. Let $F_{t}\left(w_{t}\right)$ denote the conditional distribution function of $w_{t}$ conditional on past information $\mathscr{I}_{t-1}$. Define the following function:

$$
C_{l}(q)=p_{t l}-\gamma q-\beta^{\prime} \mathrm{E}\left(x_{t} \mid \mathscr{I}_{t-1}\right) .
$$

The probability of $I_{t}=1$, given past information, is

$$
\mathrm{E}\left(I_{t} \mid \Phi_{t-1}\right)=F_{t}\left(C_{t}\left(\mathrm{E}\left(p_{t} \mid \cdot \Phi_{t-1}\right)\right)\right) \text {. }
$$

To simplify notation, denote $c_{t l}=C_{l}\left(\mathrm{E}\left(p_{t} \mid \mathscr{I}_{t-1}\right)\right)$. Eq. (2.2) can be rewritten as

$$
\begin{aligned}
p_{t} & =I_{t} p_{t l}+\left(1-I_{t}\right) p_{t}^{*} \\
& =I_{t} p_{t l}+\left(1-I_{t}\right)\left\{\gamma \mathrm{E}\left(p_{t} \mid \mathscr{I}_{t-1}\right)+\beta^{\prime} \mathrm{E}\left(x_{t} \mid \mathscr{I}_{t-1}\right)+w_{t}\right\} .
\end{aligned}
$$

Taking the conditional expectation of (2.7):

$$
\begin{aligned}
\mathrm{E}\left(p_{t} \mid \mathscr{I}_{t-1}\right)= & p_{t l} \mathrm{E}\left(I_{t} \mid \mathscr{I}_{t-1}\right)+\left\{\gamma \mathrm{E}\left(p_{t} \mid \mathscr{I}_{t-1}\right)+\beta^{\prime} \mathrm{E}\left(x_{t} \mid \mathscr{I}_{t-1}\right)\right\} \mathrm{E}\left(\left(1-I_{t}\right) \mid \mathscr{I}_{t-1}\right) \\
& +\mathrm{E}\left(\left(1-I_{t}\right) w_{t} \mid \mathscr{I}_{t-1}\right) \\
= & p_{t l} F_{t}\left(c_{t l}\right)+\left\{\gamma \mathrm{E}\left(p_{t} \mid \mathscr{I}_{t-1}\right)+\beta^{\prime} \mathrm{E}\left(x_{t} \mid \mathscr{I}_{t-1}\right)+\mathrm{E}\left(w_{t} \mid \mathscr{I}_{t-1}, I_{t}=0\right)\right\}\left[1-F_{t}\left(c_{t t}\right)\right] .
\end{aligned}
$$

Eq. (2.8) characterizes the rational expectations solution for this model. In summary, we have the following proposition.

Proposition 1. For the one-limit model, the rational expectations solution $p_{t}^{\mathrm{e}}$ is characterized by the following equation:

$$
p_{t}^{\mathrm{e}}=p_{t l} F_{t}\left(C_{l}\left(p_{t}^{\mathrm{e}}\right)\right)+\left\{\gamma p_{t}^{\mathrm{e}}+\beta^{\prime} x_{t}^{\mathrm{e}}+\mathrm{E}\left(w_{t} \mid \mathscr{I}_{t-1}, w_{t}>C_{l}\left(p_{t}^{\mathrm{e}}\right)\right)\right\}\left[1-F_{t}\left(C_{l}\left(p_{t}^{\mathrm{e}}\right)\right)\right] .
$$

The above characterization of the rational expectations solution differs from the expression in Pesaran and Samiei (1992). Pesaran and Samiei have claimed that

$$
p_{t}^{\mathrm{e}}=p_{t t} F_{t}\left(c_{t l}\right)+p_{1 t}^{\mathrm{e}}\left[1-F_{t}\left(c_{t l}\right)\right]
$$

where $p_{1 t}^{\mathrm{e}}$ was thought to be the expectation of $p_{t}$ conditional on $\mathscr{T}_{t-1}$ and $I_{t}=0$ and be given by

$$
p_{1 t}^{\mathrm{e}}=(1-\gamma)^{-1}\left\{\beta^{\prime} x_{t}^{\mathrm{e}}+\mathrm{E}\left(w_{t} \mid \mathscr{I}_{t-1}, I_{t}=0\right)\right\}
$$

[Eqs. (10) and (11) in their article]. By comparing these two equations with our Eq. (2.8), we see that the correct $p_{1 t}^{\mathrm{e}}$ should be given by

$$
p_{1 t}^{\mathrm{e}}=\gamma \mathrm{E}\left(p_{t} \mid \mathscr{I}_{t-1}\right)+\beta^{\prime} \mathrm{E}\left(x_{t} \mid \mathscr{I}_{t-1}\right)+\mathrm{E}\left(w_{t} \mid \mathscr{I}_{t-1}, I_{t}=0\right) \text {. }
$$

This relation does not imply Eq. $(*)$. This is so as follows. Taking conditional expectation to (2.1) conditional on $I_{t}=0$ and past information, it implies that 


$$
\mathrm{E}\left(p_{t} \mid \mathscr{I}_{t-1}, I_{t}=0\right)=\gamma \mathrm{E}\left(p_{t} \mid \mathscr{I}_{t-1}\right)+\beta^{\prime} \mathrm{E}\left(x_{t} \mid \mathscr{I}_{t-1}\right)+\mathrm{E}\left(w_{t} \mid \mathscr{I}_{t-1}, I_{t}=0\right),
$$

by the facts that $\mathrm{E}\left[\mathrm{E}\left(p_{t} \mid \mathscr{I}_{t-1}\right) \mid \mathscr{I}_{t-1}, I_{t}=0\right]=\mathrm{E}\left(p_{t} \mid \mathscr{I}_{t-1}\right)$ and $\mathrm{E}\left[\mathrm{E}\left(x_{t} \mid \mathscr{I}_{t-1}\right) \mid \mathscr{I}_{t-1}, I_{t}=0\right]=$ $\mathrm{E}\left(x_{t} \mid \mathscr{I}_{t-1}\right)$. The $p_{1 t}^{\mathrm{e}}$ is $\mathrm{E}\left(p_{t} \mid \mathscr{I}_{t-1}, I_{t}=0\right)$. The $p_{1 t}^{\mathrm{e}}$ could be written as $(*)$ if and only if $\mathrm{E}\left(p_{t} \mid \mathscr{I}_{t-1}, I_{t}=0\right)$ were equal to $\mathrm{E}\left(p_{t} \mid \mathscr{I}_{t-1}\right)$ in (2.9). But these two quantities will not be equal to each other in a limited dependent variables model because $I_{t}=0$ provides extra information about $p_{t}$ in addition to the past information as $I_{t}$ is not in the formation set $\mathscr{I}_{t-1}$.

Our analysis can be easily generalized to two-limit models. A two-limit model is for the case where the dependent variable is bounded from above and below; say, respectively, by $p_{t u}$ and $p_{t l}$, where $p_{t u}>p_{t l}$. The observed dependent variable $p_{t}$ at time $t$ will be

$$
p_{t}=\min \left\{p_{t u}, \max \left\{p_{t}^{*}, p_{t l}\right\}\right\},
$$

where $p_{t}^{*}$ is defined in (2.1). The bounds $p_{t l}$ and $p_{t u}$ are known at time $t-1$ and are in the information set $\mathscr{I}_{t-1}$. This model generalizes the one-limit case and has interesting application in models of exchange rate determination (Pesaran and Samiei, 1992). Define dichotomous indicators $I_{1 t}, I_{2 t}$ and $I_{3 t}$ to represent the three different regimes: $I_{1 t}=1$ if $p_{t}^{*} \leqslant p_{t l}, I_{2 t}=1$ if $p_{t}^{*} \geqslant p_{t u}$, and $I_{3 t}-1$ if $p_{t l}<p_{t}^{*}<p_{t u}$. In addition to the function $C_{l}$ in (2.5) and $c_{t l}$ defined before, define

$$
C_{u}(q)=p_{t u}-\gamma q-\beta^{\prime} x_{t}^{\mathrm{e}},
$$

and $c_{t u}=C_{u}\left(p_{t}^{\mathrm{e}}\right)$. The regime probabilities are that $\mathrm{E}\left(I_{1 t} \mid \mathscr{F}_{t-1}\right)=F_{t}\left(c_{t l}\right), \mathrm{E}\left(I_{2 t} \mid \mathscr{I}_{t-1}\right)=1-$ $F_{t}\left(c_{t u}\right)$, and $\mathrm{E}\left(I_{3 t} \mid \mathscr{I}_{t-1}\right)=F_{t}\left(c_{t u}\right)-F_{t}\left(c_{t l}\right)$. Eq. (2.10) can be rewritten as $p_{t}=I_{1 t} p_{t l}+I_{2 t} p_{t u}+$ $I_{3 t} p_{t}^{*}$. It follows that

$$
\begin{aligned}
\mathrm{E}\left(p_{t} \mid \mathscr{I}_{t-1}\right)= & p_{t t} F_{t}\left(c_{t l}\right)+p_{t u}\left[1-F_{t}\left(c_{t u}\right)\right] \\
& +\left\{\gamma \mathrm{E}\left(p_{t} \mid \mathscr{I}_{t-1}\right)+\beta^{\prime} \mathrm{E}\left(x_{t} \mid \mathscr{I}_{t-1}\right)+\mathrm{E}\left(w_{t} \mid \mathscr{I}_{t-1}, I_{3 t}=1\right)\right\}\left[F_{t}\left(c_{t u}\right)-F_{t}\left(c_{t l}\right)\right] .
\end{aligned}
$$

Therefore, for this model, we have the following characterization of the rational expectations solution.

Proposition 2. For the two-limit model, the rational expectations solution $p_{t}^{\mathrm{e}}$ is characterized by the following equation:

$$
\begin{aligned}
p_{t}^{\mathrm{e}}= & p_{t l} F_{t}\left(C_{l}\left(p_{t}^{\mathrm{e}}\right)\right)+p_{t u}\left[1-F_{t}\left(C_{u}\left(p_{t}^{\mathrm{e}}\right)\right)\right] \\
& +\left\{\gamma p_{t}^{\mathrm{e}}+\beta^{\prime} x_{t}^{\mathrm{e}}+\mathrm{E}\left(w_{t} \mid \mathscr{I}_{t-1}, C_{l}\left(p_{t}^{\mathrm{e}}\right)<w_{t}<C_{u}\left(p_{t}^{\mathrm{e}}\right)\right)\right\}\left[F_{t}\left(C_{u}\left(p_{t}^{\mathrm{e}}\right)\right)-F_{t}\left(C_{l}\left(p_{t}^{\mathrm{e}}\right)\right)\right] .
\end{aligned}
$$

By the same reasoning, the characterization of the rational expectations solution for the two-limit model in Pesaran and Samiei (1992, Eq. (23)) differs from the above one. The above equation provides the correct characterization of the solution. For the two-limit model, Donald and Maddala (1992) provided the first correct characterization of the solution under the assumptions that the disturbances are serially independent and are normally distributed (see Eq. (20) in Donald and Maddala, 1992). The characterization in Donald and Maddala is valid only if $\gamma \neq 1$. Our characterizations in both Propositions 1 and 2 are general without such assumptions. 


\section{The existence and uniqueness of the rational expectations solution}

In this section we consider the problem of existence of the rational expectations solution. It is convenient to first consider the two-limit model and then the onc-limit model. In the proofs, we assume that the absolute first (conditional) moment $\mathrm{E}\left(|w| \mid \mathscr{I}_{t-1}\right)$ of $w$ exists and the conditional distribution $F_{t}(w)$ of $w$ is absolutely continuous with an everywhere positive (conditional) density function $f_{t}(w)$. This will include in particular the normal distribution case considered in Pesaran and Samiei (1992) and Donald and Maddala (1992). Their normality assumption has implicitly ruled out the possibility of serially correlated disturbances. This is so because if the disturbances are serially correlated, the conditional distribution of the disturbances conditional on past information cannot be normally distributed in limited dependent variables models.

Proposition 3. For the two-limit model, the rational expectations solution for $p_{t}^{\mathrm{e}}$ exists for any value of $\gamma$. If $\gamma \leqslant 1$, the solution is unique.

Proof. Define a function $G$ :

$$
\begin{aligned}
G(q)= & p_{t l} F_{t}\left(C_{l}(q)\right)+p_{t u}\left[1-F_{t}\left(C_{u}(q)\right)\right] \\
& +\left\{\gamma q+\beta^{\prime} x_{t}^{\mathrm{e}}+\mathrm{E}\left(w_{t} \mid g_{t-1}, C_{l}(q)<w_{t}<C_{u}(q)\right)\right\}\left[F_{t}\left(C_{u}(q)\right)-F_{t}\left(C_{l}(q)\right)\right] .
\end{aligned}
$$

For any value $q$, consider the random variable $z^{*}$ defined by

$$
z^{*}=\gamma q+\beta^{\prime} x_{t}^{e}+w_{t} .
$$

This is an artificial random variable. For this $z^{*}$, it is easy to see that

$$
\mathrm{E}\left(z^{*} \mid \mathscr{I}_{t-1}, p_{t l}<z^{*}<p_{t u}\right)=\gamma q+\beta^{\prime} x_{t}^{\mathrm{e}}+\mathrm{E}\left(w_{t} \mid \mathscr{I}_{t-1}, C_{l}(q)<w_{t}<C_{u}(q)\right) .
$$

Since, for any constants $a$ and $b$, with $a<b, \mathrm{E}\left(z^{*} \mid a<z^{*}<b\right)$ must lie between $a$ and $b$, this demonstrates the following inequality:

$$
p_{t l}<\left\{\gamma q+\beta^{\prime} x_{t}^{\mathrm{e}}+\mathrm{E}\left(w_{t} \mid \mathscr{I}_{t-1}, C_{l}(q)<w_{t}<C_{u}(q)\right)\right\}<p_{t u},
$$

for any value $q$. Since $p_{t}<p_{t u}$, it follows that $G(q)<p_{t u} F_{t}\left(C_{l}(q)\right)+p_{t u}\left[1-F_{t}\left(C_{u}(q)\right)\right]+$ $p_{t u}\left[F_{t}\left(C_{u}(q)\right)-F_{t}\left(C_{l}(q)\right)\right]=p_{t u}$ and $G(q)>p_{t l}$. In particular, $G\left(p_{t l}\right)>p_{t l}$ and $G\left(p_{t u}\right)<p_{t u}$. Consider the function $H(q)=q-G(q)$. It follows that $H\left(p_{t l}\right)<0$ and $H\left(p_{t u}\right)>0$. Since $H(q)$ is continuous, it must have a root inside the interval $\left(p_{t l}, p_{t u}\right)$. This proves that the rational expectations solution exists.

Let $f_{t}\left(w_{t}\right)$ denote the density function of $w_{t}$ given $\mathscr{I}_{t-1}$. It follows that

$$
\mathrm{E}\left(w_{t} \mid g_{t-1}, C_{l}(q)<w_{t}<C_{u}(q)\right)=\int_{C_{l}(q)}^{C_{u}(q)} w f_{t}(w) \mathrm{d} w /\left[F_{t}\left(C_{u}(q)\right)-F_{t}\left(C_{l}(q)\right)\right],
$$

and hence 


$$
\begin{aligned}
G(q)= & p_{t l} F_{t}\left(C_{l}(q)\right)+p_{t u}\left[1-F_{t}\left(C_{u}(q)\right)\right]+\left(\gamma q+\beta^{\prime} x_{t}^{\mathrm{e}}\right)\left[F_{t}\left(C_{u}(q)\right)-F_{t}\left(C_{l}(q)\right)\right] \\
& +\int_{C_{l}(q)}^{C_{u}(q)} w f_{t}(w) \mathrm{d} w .
\end{aligned}
$$

Differentiating the function $G$ from the above equation,

$$
\begin{aligned}
\frac{\mathrm{d} G(q)}{\mathrm{d} q}= & (-\gamma)\left\{p_{t l} f_{t}\left(C_{l}(q)\right)-p_{t u} f_{t}\left(C_{u}(q)\right)+\left(\gamma q+\beta^{\prime} x_{t}^{\mathrm{e}}\right)\left[f_{t}\left(C_{u}(q)\right)-f_{t}\left(C_{l}(q)\right)\right]\right. \\
& \left.\left.+\left[C_{u}(q) f_{t}\left(C_{u}(q)\right)-C_{l}(q)\right) f_{t}\left(C_{l}(q)\right)\right]\right\}+\gamma\left[F_{t}\left(C_{u}(q)\right)-F_{t}\left(C_{l}(q)\right)\right] \\
= & \gamma\left[F_{t}\left(C_{u}(q)\right)-F_{l}\left(C_{l}(q)\right)\right]
\end{aligned}
$$

where the second equality follows from (2.5) and (2.11). It follows that $\mathrm{d} H(q) / \mathrm{d} q=1-$ $\gamma\left[F_{t}\left(C_{u}(q)\right)-F_{t}\left(C_{l}(q)\right)\right]$. For $\gamma \leqslant 1, \mathrm{~d} H(q) / \mathrm{d} q>0$ that implies the solution is unique.

Q.E.D.

The existence of the rational expectations solution in the two-limit model is general without any restriction on the parameter $\gamma$. This corrects the claim in Pesaran and Samiei (1992, Proposition 2) which states that, if $\gamma$ is greater than unity, a solution may not exist, and for $\gamma$ equal to one no solution will exist. The mistaken conclusion is due to the analysis of the incorrect characterization equation of the rational expectations of this model. It generalizes also the result in Donald and Maddala (1992) which states that there exists a unique solution if $\gamma<1$. The solution exists and is unique, even for $\gamma=1$.

The sufficient condition on $\gamma$ for the uniqueness solution can be slightly extended. The $\mathrm{d} H(q) / \mathrm{d} q$ can still be strictly positive on the interval $\left[p_{t l}, p_{t u}\right]$ under the condition

$$
\gamma<\left\{\max _{p_{l} \leqslant q \leqslant p_{u}}\left[F_{t}\left(C_{u}(q)\right)-F_{t}\left(C_{l}(q)\right)\right]\right\}^{-1} .
$$

Dependent on $F_{t}$ and the values of variables in the model, it is possible to have a unique solution when $\gamma$ is greater than one. It is difficult to have more general results for the $\gamma>1$ case because $H(q)$ might no longer be a monotonic increasing function. By taking the second-order derivatives of $H(q)$, we see that

$$
\frac{\mathrm{d}^{2} H(q)}{\mathrm{d} q^{2}}=\gamma^{2}\left[f_{t}\left(C_{u}(q)\right)-f_{t}\left(C_{l}(q)\right)\right] \text {. }
$$

If $f_{t}$ is a unimodal density function, for a positive value of $\gamma, H(q)$ might have a concave section and then change into a convex shape as $q$ becomes large. The $H(q)$ with such a shape might cross the axis more than once as it changes from a negative $H\left(p_{t l}\right)$ to a positive value $H\left(p_{t u}\right)$.

Proposition 4. For the one-limit model, the rational expectations solution $p_{t}^{\mathrm{e}}$ exists and is unique for $\gamma<1$. For $\gamma=1$, if a solution exists, it is unique. However, in general, there are either multiple solutions or no solution when $\gamma>1$.

Proof. The one-limit case corresponds to $p_{t u}$ being positive infinity in the two-limit case. For this case, 


$$
H(q)=q-p_{t l} F_{t}\left(C_{l}(q)\right)-\left(\gamma q+\beta^{\prime} x_{t}^{e}\right)\left[1-F_{t}\left(C_{l}(q)\right)\right]-\int_{C_{l}(q)}^{\infty} w f_{t}(w) \mathrm{d} w
$$

Furthermore,

$$
\frac{\mathrm{d} H(q)}{\mathrm{d} q}=1-\gamma\left[1-F_{t}\left(C_{l}(q)\right)\right],
$$

and $H\left(p_{t l}\right)=p_{t l}-G\left(t_{t l}\right)<0$ because $\gamma q+\beta^{\prime} x_{t}^{\mathrm{e}}+\mathrm{E}\left(w_{t} \mid \mathscr{I}_{t-1}, C_{l}(q)<w_{t}\right)>p_{t l}$.

It remains to investigate the limiting behavior of $H(q)$ as $q$ tends to positive infinity. $H(q)$ can be written as a difference of two terms: $H(q)=H_{1}(q)-H_{2}(q)$, where

$$
H_{1}(q)=\left(1-\gamma\left[1-F_{t}\left(C_{l}(q)\right)\right]\right)_{q}
$$

and

$$
H_{2}(q)=p_{t l} F_{t}\left(C_{l}(q)\right)+\beta^{\prime} x_{t}^{\mathrm{e}}\left[1-F_{t}\left(C_{l}(q)\right)\right]+\int_{C_{l}(q)}^{\infty} w f_{t}(w) \mathrm{d} w .
$$

As $\mathrm{E}\left(|w| \mid \mathscr{I}_{t-1}\right)<\infty$, the second term is apparently bounded in $q$.

Case (i): $\gamma<1$. It is easy to check that for $\gamma<1, \lim _{q \rightarrow+\infty} H_{1}(q)=+\infty$ by using the property that when $\gamma>0, \lim _{q \rightarrow+\infty} F_{t}\left(C_{l}(q)\right)=0$. Also, $\mathrm{d} H(q) / \mathrm{d} q>0$ for all $q$ when $\gamma<1$. Therefore, as $H(q)$ is strictly increasing and it will definitely cross the axis, $H(q)=0$ has a solution and the solution is unique.

Case (ii): $\gamma=1$. For this case, $H(q)$ is still a strictly increasing function as $\mathrm{d} H(q) / \mathrm{d} q=$ $F_{t}\left(C_{l}(q)\right)>0$. But $\quad \lim _{q \rightarrow+\infty} H(q)=\lim _{q \rightarrow+\infty} q F_{t}\left(C_{l}(q)\right)-\beta^{\prime} x_{t}^{\mathrm{e}}=\lim _{q \rightarrow+\infty} q^{2} f_{t}\left(C_{l}(q)\right)-\beta^{\prime} x_{t}$ which may be positive or negative. If the limiting value is positive, the solution will exist; otherwise, no solution will exist.

Case (iii): $\quad \gamma>1$. For this case, $\lim _{q \rightarrow+\infty} H_{1}(q)=(1-\gamma) \lim _{q \rightarrow+\infty} q=-\infty$. Also $\lim _{q \rightarrow+\infty} \mathrm{d} H(q) / \mathrm{d} q=1-\gamma<0$. For $\gamma \geqslant 1 /\left[1-F_{t}\left(c_{t l}\right)\right], \mathrm{d} H(q) / \mathrm{d} q \leqslant 0$ for all $q \geqslant p_{t l}$ and no solution will exist. When $\gamma<1 /\left[1-F_{t}\left(c_{t l}\right)\right]$, the function $H(q)$ will increase around $p_{t l}$ but will eventually decrease to $-\infty$. The maximum of $H(q)$ occurs at $\bar{q}=\left\{p_{t l}-\beta^{\prime} x_{t}^{\mathrm{e}}-F_{t}^{-1}(1-1 / \gamma)\right\} /$ $\gamma$, which is solved from the first-order condition $1-\gamma\left[1-F_{l}\left(C_{l}(\bar{q})\right)\right]=0$. The maximum is

$$
H(\bar{q})=-\beta^{\prime} x_{t}^{\mathrm{e}} / \gamma-(1-1 / \gamma) p_{t l}-\int_{F_{t}^{-1}(1-1 / \gamma)}^{\infty} w f_{t}(w) \mathrm{d} w .
$$

If $H(\bar{q})$ is strictly positive, there are two solutions. If $H(\bar{q})$ is strictly negative, there will be no solution. The case that $H(\bar{q})$ is exactly zero is a rare case [probability zero if $H(\bar{q})$ is a continuous random variable]. Q.E.D.

From these results we see that for the limited dependent variables model (2.1) with either one or two limits, rational expectations can be well defined as long as the values of $\gamma$ are not greater than one. Some behavioral models may impose theoretical restrictions on possible values of $\gamma$. For example, the price support commodity market model in Shonkwiler and Maddala (1985) is a two-equation model with a supply equation $S_{t}$ and a demand equation $D_{t}$ : 


$$
\begin{aligned}
& S_{t}=\alpha_{1} p_{t}^{\mathrm{c}}+\beta_{1}^{\prime} x_{t}+\epsilon_{1 t}, \\
& D_{t}=\alpha_{2} p_{t}^{*}+\beta_{2}^{\prime} x_{t}+\epsilon_{2 t} .
\end{aligned}
$$

The reduced-form price cquation implicd by these cquations is $p_{t}^{*}=\gamma p_{t}^{\mathrm{e}} \mid \beta^{\prime} x_{t}+u_{t}$ with $\gamma=\alpha_{1} / \alpha_{2}$. As $\alpha_{1}$ in the supply equation is expected to be positive and $\alpha_{2}$ negative in the demand equation, the implied $\gamma$ will be negative. Thus for the price support commodity market model, a rational expectations equilibrium of the market price can exist and is unique for each period.

\section{Acknowledgments}

The author gratefully acknowledges support from the National Science Foundation under grant SBR-9223325 for his research. I appreciate valuable comments from Professors G.S. Maddala and M.H. Pesaran on this paper.

\section{References}

Amemiya, T., 1973, Regression analysis when the dependent variable is truncated normal, Econometrica 41, 997-1016.

Chanda, A.K. and G.S. Maddala, 1983, Methods of estimation for models of markets with bounded price variation under rational expectations, Economics Letters 13, 181-184. Erratum in: 1984, Economics Letters 15, 195-196.

Donald, S.G. and G.S. Maddala, 1992, A note on the estimation of limited dependent variables models under rational expectations, Economics Letters 38, 17-23.

Holt, M.T. and S.R. Johnson, 1989, Bounded price variation and rational expectations in an endogenous switching model of the US corn market, Review of Economics and Statistics 71, 605-613.

Maddala, G.S., 1990, Estimation of dynamic disequilibrium models with rational expectations: The case of the commodity markets, in: L.A. Winters and D. Sapsford, eds., Primary commodity prices: Economic models and policy (Cambridge University Press, Cambridge).

Muth, J.F., 1961, Rational expectations and the theory of price movements, Econometrica 29, 315-335.

Pesaran, M.H., 1990, Comment on Maddala's paper, in: L.A. Winters and D. Sapsford, eds., Primary commodity prices: Economic models and policy (Cambridge University Press, Cambridge).

Pcsaran, M.H. and F.J. Rugc-Murcia, 1993, Limited-dependent rational expectation models with stochastic thresholds, manuscript, Department of Applied Economics, University of Cambridge.

Pesaran, M.H. and H. Samiei, 1992, Estimating limited-dependent rational expectations models with an application to exchange rate determination in a target zone, Journal of Econometrics 53, 141-163.

Shonkwiler, J.S. and G.S. Maddala, 1985, Modelling expectations of bounded prices: An application to the market for corn, Revicw of Economics and Statistics 67, 634-641.

Tobin, J., 1958, Estimation of relationships for limited dependent variables, Econometrica 26, 24-36. 